\title{
A New Approach to Modeling the Nucleation and Growth Kinetics of Tricalcium Silicate Hydration
}

\author{
Jeffrey J. Thomas ${ }^{\dagger}$ \\ Department of Civil and Environmental Engineering, Northwestern University, Evanston, Illinois 60208
}

\begin{abstract}
The hydration kinetics of tricalcium silicate $\left(\mathrm{C}_{3} \mathrm{~S}\right)$, the main constituent of portland cement, were analyzed with a mathematical "boundary nucleation" model in which nucleation of the hydration product occurs only on internal boundaries corresponding to the $\mathrm{C}_{3} \mathrm{~S}$ particle surfaces. This model more closely approximates the $\mathrm{C}_{3} \mathrm{~S}$ hydration process than does the widely used Avrami nucleation and growth model. In particular, the boundary model accounts for the important effect of the $\mathrm{C}_{3} \mathrm{~S}$ powder surface area on the hydration kinetics. Both models were applied to isothermal calorimetry data from hydrating $\mathrm{C}_{3} \mathrm{~S}$ pastes in the temperature range of $10^{\circ}-40^{\circ} \mathrm{C}$. The boundary nucleation model provides a better fit to the early hydration rate peak than does the Avrami model, despite having one less varying parameter. The nucleation rate (per unit area) and the linear growth rate of the hydration product were calculated from the fitted values of the rate constants and the independently measured powder surface area. The growth rate follows a simple Arrhenius temperature dependence with a constant activation energy of $31.2 \mathrm{~kJ} / \mathrm{mol}$, while the activation energy associated with the nucleation rate increases with increasing temperature. The start of the nucleation and growth process coincides with the time of initial mixing, indicating that the initial slow reaction period known as the "induction period" is not a separate chemical process as has often been hypothesized.
\end{abstract}

\section{Introduction}

$\mathrm{T}$ HE usefulness of concrete made from portland cement concrete results to a large extent from the ability to control the initial rate of reaction between cement and water so that freshly mixed concrete remains fluid and workable as it is being placed and then sets shortly thereafter. Altering the kinetics of this process, either to extend the period of workability or shorten the time to set, is one of the most important functions of the many chemical admixtures that are routinely added to concrete. Despite the fundamental importance of this process to the construction industry, and the fact that rate-controlling chemical admixtures are of significant industrial importance, the fundamental mechanisms underlying the reaction process are not well understood, and there is no single theory that can explain all of the major observations associated with the early hydration of portland cement.

Tricalcium silicate $\left(3 \mathrm{CaO} \cdot \mathrm{SiO}_{2}\right.$, or $\left.\mathrm{C}_{3} \mathrm{~S}^{\ddagger}\right)$ is the main mineral constituent of portland cement, and its hydration forms most of the new solid phases that give hardened cement paste its strength. A comprehensive review of $\mathrm{C}_{3} \mathrm{~S}$ hydration is given by

M. Grutzeck—contributing editor

Manuscript No. 22597. Received December 20, 2006; approved March 20, 2007. Some of the results in this paper were presented by the author in a technical symposium at the Materials Science \& Technology Meeting, October 16, 2006, in Cincinnati, OH.

This work was supported by the National Science Foundation under contract CMS 0409571 edu

Author to whom correspondence should be addressed. e-mail: jthomas@northwestern. ${ }^{\ddagger}$ Cement chemistry notation: $\mathrm{C}=\mathrm{CaO}, \mathrm{S}=\mathrm{SiO}_{2}, \mathrm{H}=\mathrm{H}_{2} \mathrm{O}$.
Gartner et al. ${ }^{1}$ Pure $\mathrm{C}_{3} \mathrm{~S}$ is often used as a model system for portland cement, because the hydration kinetics and the properties of the hardened paste are quite similar. Hydration of $\mathrm{C}_{3} \mathrm{~S}$ occurs via a dissolution-precipitation reaction that forms calcium-silicate-hydrate $(\mathrm{C}-\mathrm{S}-\mathrm{H})$ gel and calcium hydroxide, which can be approximated as

$$
\begin{aligned}
3 \mathrm{CaO} \cdot \mathrm{SiO}_{2}+5.3 \mathrm{H}_{2} \mathrm{O} \Rightarrow & 1.7 \mathrm{CaO}-\mathrm{SiO}_{2}-4 \mathrm{H}_{2} \mathrm{O} \\
& +1.3 \mathrm{Ca}(\mathrm{OH})_{2}
\end{aligned}
$$

The $\mathrm{C}-\mathrm{S}-\mathrm{H}$ gel phase is a precipitated colloid with a high specific surface area and a nanometer-scale system of internal gel pores, ${ }^{2}$ and the formula shown in Eq. (1) includes liquid water trapped in nanopores that is no longer available for hydration. Equation (1) is exothermic, and the most accurate method of measuring the kinetics is to monitor the rate of heat output with isothermal calorimetry. Figure 1 shows the calorimetry results for a $\mathrm{C}_{3} \mathrm{~S}$ paste hydrated at $20^{\circ} \mathrm{C}$, with the main features ${ }^{3,4}$ of the reaction kinetics labeled. $\mathrm{C}_{3} \mathrm{~S}$ has a very high theoretical solubility, and thus when $\mathrm{C}_{3} \mathrm{~S}$ and water are combined, there is a short $(<1 \mathrm{~min})$ period of fast reaction and heat output as the $\mathrm{C}_{3} \mathrm{~S}$ dissolves (stage 1). Stage 2 is a period of slow reaction commonly termed the induction period. The end of stage 1 is usually attributed ${ }^{4,5}$ to the formation of a thin hydrated layer on the $\mathrm{C}_{3} \mathrm{~S}$ particles that acts as a diffusion barrier to dissolving ions, but another school of thought ${ }^{6}$ holds that there is no protective layer and that the observed kinetics are explained fully by the difficulty in nucleating $\mathrm{C}-\mathrm{S}-\mathrm{H}$ gel.

During the next period of reaction (stage 3 in Fig. 1), called the acceleratory period, the rate of reaction increases continuously, reaching a maximum at a time that is usually $<24 \mathrm{~h}$ after initial mixing. Both the maximum rate and the time at which it occurs depend strongly on the temperature and on the particle size distribution of the $\mathrm{C}_{3} \mathrm{~S}$. The reaction rate then decreases rapidly to less than half of its maximum value (stage 4 ), and then decreases much more slowly until all of the $\mathrm{C}_{3} \mathrm{~S}$ or all of the water is consumed, or all of the space available for reaction products is filled (stage 5), which can take weeks or months. The early hydration period is generally considered to comprise stages $1-4$.

Despite the greater simplicity of the $\mathrm{C}_{3} \mathrm{~S}-\mathrm{H}_{2} \mathrm{O}$ system as compared with portland cement, the kinetics of $\mathrm{C}_{3} \mathrm{~S}$ hydration are not fully understood. There is significant evidence that the early hydration period occurs by a nucleation and growth process, and kinetic data from $\mathrm{C}_{3} \mathrm{~S}$ hydration are frequently fitted to the standard Avrami-type nucleation and growth equation, as this provides a satisfactory fit to a limited portion of the data in the vicinity of the rate peak ${ }^{7-15}$ (see Fig. 1). However, the resulting fit parameters are difficult to interpret, and it is generally recognized $^{5,16}$ that the $\mathrm{C}_{3} \mathrm{~S}$ hydration process, in which the hydration product grows outward from the $\mathrm{C}_{3} \mathrm{~S}$ particle surfaces into the surrounding water-filled pores, violates the conditions under which the standard Avrami equation is derived. In this paper, an alternative set of nucleation and growth equations, developed originally to describe the kinetics of a solid phase 


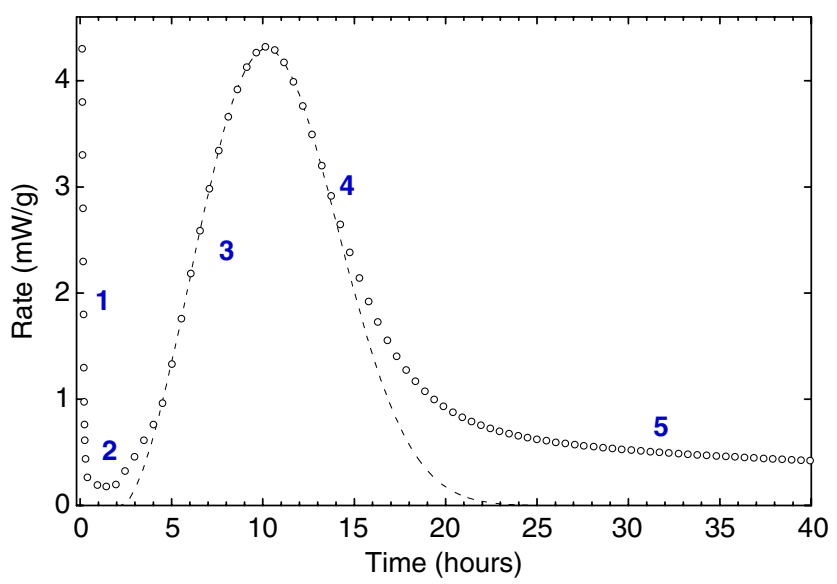

Fig. 1. Rate of heat evolution from a $\mathrm{C}_{3} \mathrm{~S}$ paste mixed at a water/cement ratio of 0.5 by mass and hydrated at $20^{\circ} \mathrm{C}(\mathrm{O})$. The kinetic stages labeled 1-5 are discussed in the text. The dashed line represents the fit obtained by applying a version of the standard Avrami nucleation and growth equation (Eq. (9)) to the data. The fit parameters are given in Table II.

change nucleated preferentially at grain boundaries, ${ }^{17}$ is shown to provide a better description of $\mathrm{C}_{3} \mathrm{~S}$ hydration.

\section{Mathematical Nucleation and Growth Models}

\section{(1) The Standard Avrami Nucleation and Growth Model}

For the purposes of this paper, it is useful to revisit the derivation of the standard Avrami equation. Equations (2)-(7) below are based on the original work of Avrami, ${ }^{18,19}$ Kolmogorov, ${ }^{20}$ and Johnson and Mehl, ${ }^{21}$ and a more complete derivation can be found in Christian. ${ }^{22}$ Consider an isothermal phase transformation that occurs within a fixed volume by a nucleation and growth process for which nucleation is spatially random and the linear growth rate $G$ in any direction is constant. Each region of transformed phase will be spherical, with volume $4 \pi / 3 G^{3}(t-\tau)^{3}$, where $t$ is time since the start of the transformation and $\tau$ is the time at which the particular region nucleated. The "extended" transformed volume fraction $X^{\mathrm{e}}$, which ignores any interference between growing regions, is found by summing the contributions of the individual regions:

$$
X^{\mathrm{e}}=\frac{4 \pi}{3} \int_{\tau=0}^{t} I_{\mathrm{v}} G^{3}(t-\tau)^{3} \mathrm{~d} \tau
$$

where $I_{\mathrm{v}}$ is the rate of nucleation per unit of untransformed volume. Alternatively, if all nuclei are already present at the start of the transformation, or if a fixed number of nuclei form rapidly at the start of the reaction (a situation called site saturation), the extended volume fraction is simply

$$
X^{\mathrm{e}}=\frac{4 \pi N_{\mathrm{v}}^{0}}{3} G^{3} t^{3}
$$

where $N_{\mathrm{v}}^{0}$ is the number of nuclei per unit volume.

In the later stages of the transformation, the growing regions will impinge on each other, forming a common interface over which there is no growth. Provided that the probability of nucleation is the same everywhere in the untransformed volume, the relationship between the true and extended transformed volume fractions is

$$
X^{\mathrm{e}}=-\ln (1-X)
$$

If the nucleation rate within the untransformed volume, $I_{\mathrm{v}}$, is taken to be constant with time, then combining Eqs. (2) and (4) gives

$$
X=1-\exp \left(\frac{-\pi G^{3} I_{\mathrm{v}}}{3} t^{4}\right)
$$

while for site saturation, combining Eqs. (3) and (4) gives

$$
X=1-\exp \left(-\frac{4 \pi G^{3} N_{\mathrm{v}}^{0}}{3} t^{3}\right)
$$

As proposed by Avrami, ${ }^{18,19}$ these limiting cases can be combined into a single general equation

$$
X=1-\exp \left[-\left(k_{\mathrm{avr}} t\right)^{n}\right]
$$

where $k_{\text {avr }}$ is an effective rate constant. If nucleation is heterogeneous, such that the rate of nucleation at preferred sites (also distributed randomly through the volume) decreases with time, then the exponent $n$ in Eq. (7) will lie between the limiting values of 3 and 4 . The rate of transformation is obtained by differentiating Eq. (7) with respect to time:

$$
\frac{\mathrm{d} X}{\mathrm{~d} t}=n k_{\mathrm{avr}}^{n} t^{n-1} \exp \left(-\left(k_{\mathrm{avr}} t\right)^{n}\right)
$$

For direct application to rate data for $\mathrm{C}_{3} \mathrm{~S}$ hydration, such as that obtained from isothermal calorimetry, it is necessary to use a modified version of Eq. (8):

$$
R=A n k_{\mathrm{avr}}^{n}\left(t-t_{0}\right)^{n-1} \exp \left(-\left[k_{\mathrm{avr}}\left(t-t_{0}\right)\right]^{n}\right)
$$

where $R$ is the hydration rate, $A$ is a normalization constant, and $t_{0}$ is a delay between the time of mixing and the time of start of nucleation and growth kinetics. Equation (9) represents a fourparameter model, as $A, k_{\mathrm{avr}}, t_{0}$, and $n$ are varied. If the measured hydration rate $R$ is normalized by the total amount of hydration that occurs when all of the $\mathrm{C}_{3} \mathrm{~S}$ is consumed, then the parameter $A$ can be interpreted as the fraction of $\mathrm{C}_{3} \mathrm{~S}$ hydration that occurs by nucleation and growth.

When applying the Avrami model to a chemical reaction (such as $\mathrm{C}_{3} \mathrm{~S}$ hydration) rather than a phase transformation, it is implicitly assumed that the rate of diffusion of reactants to the growing surface is not rate controlling. While diffusion rates through the bulk solution are much faster than the $\mathrm{C}_{3} \mathrm{~S}$ hydration rate, this point becomes important at later times when the reactants must diffuse through the nanopores within a continuous product layer around the unhydrated particles. This issue applies to the boundary nucleation and growth model described in the next section as well. The fitted values of parameter $A$ from both models indicate that more than half of the hydration reaction is controlled by diffusion rather than by nucleation and growth (stage 5).

Reported measured values of $n$ for $\mathrm{C}_{3} \mathrm{~S}$ hydration vary widely, ${ }^{7,8,10,11-14}$ with the majority falling between 2 and $3,^{11}$ which is outside the theoretical range of 3-4 noted above. This is often justified by suggesting that the formation of the hydration product occurs with a dimensionality of $<3.8,12,13,23$ This may well be the case at the nanometer scale, as the nanometer-scale morphology of the $\mathrm{C}-\mathrm{S}-\mathrm{H}$ gel phase has been variously described as fibrillar, ${ }^{24}$ sheetlike, ${ }^{25}$ or globular. ${ }^{26}$ However, at the larger scale of the capillary pore system, the average density, including internal gel pores, of the product that forms during the early hydration period (primarily the low-density morphology of $\mathrm{C}-\mathrm{S}-\mathrm{H}$ gel) exhibits a statistically random distribution around a characteristic value. ${ }^{27}$ In this case, for the purposes of describing the overall kinetics of the system, the dimensionality of the growth should be close to 3, regardless of the nanometer-scale morphology of the solid $\mathrm{C}-\mathrm{S}-\mathrm{H}$ gel phase. It will be shown in a later section that when the standard Avrami model is used to fit the data only up to the rate peak, the fitted value of $n$ is close to 4 , corresponding to three-dimensional growth and a constant nucleation rate. 


\section{(2) Boundary Nucleation and Growth Model}

A serious objection to the use of Eqs. (8) and (9) to model $\mathrm{C}_{3} \mathrm{~S}$ hydration is the assumption that nucleation occurs at randomly distributed locations within the untransformed volume, which is required in order for the relationship between the true and extended transformed volume fractions given by Eq. (4) to hold. For the hydration of $\mathrm{C}_{3} \mathrm{~S}$ (and also portland cement), it is well established ${ }^{5}$ that hydration product forms first on the surface of the particles. The hydration product grows along the surface of the particles, eventually covering them, and also outward into the pore space between the particles, so that product regions from adjacent particles eventually coalesce, causing the paste to set. This process is analogous to a phase transformation in a polycrystalline solid that is nucleated preferentially at the grain boundaries. The kinetics of nucleation and growth under the conditions of grain boundary nucleation were derived mathematically by Cahn, ${ }^{17}$ and these equations will be applied here to $\mathrm{C}_{3} \mathrm{~S}$ hydration. The derivation of Eqs. (10)-(14) in this section is also based on the discussion of Cahn's original work by Christian. $^{22}$

Consider an untransformed volume containing single planar boundary, and assume that nucleation of the transformed phase occurs only at spatially random locations on this boundary. Now consider a plane parallel to the boundary, at a perpendicular distance $y$. The intersection of a single growing spherical region of $\beta$-phase nucleated at time $\tau$ and this plane is a circle with area $\pi\left(G^{2}(t-\tau)^{2}-y^{2}\right)$ if $t>\tau+y / G$. (If $t<\tau+y / G$, the region of transformed phase has not yet reached the plane and the area is zero.)

The extended area fraction of the intersection between the plane and all regions nucleated on the grain boundary, denoted $Y^{\mathrm{e}}$, is

$$
\begin{array}{ll}
Y^{\mathrm{e}}=\pi \int_{0}^{t-\frac{y}{G}} I_{\mathrm{B}}\left(G^{2}(t-\tau)^{2}-y^{2}\right) \mathrm{d} \tau & (\text { if } t>y / G) \\
Y^{\mathrm{e}}=0 & \text { (if } t<y / G)
\end{array}
$$

where $I_{\mathrm{B}}$ is the nucleation rate per unit area of untransformed boundary. If $I_{\mathrm{B}}$ is assumed to be constant, Eq. (10) can be integrated directly, resulting in in $^{\S}$

$$
\begin{array}{ll}
Y^{\mathrm{e}}=\frac{\pi I_{\mathrm{B}}}{3} G^{2} t^{3}\left[1-\frac{3 y^{2}}{G^{2} t^{2}}+\frac{2 y^{3}}{G^{3} t^{3}}\right] & (\text { if } t>y / G) \\
Y^{\mathrm{e}}=0 & (\text { if } t<y / G)
\end{array}
$$

With the assumption that nucleation sites are randomly distributed on the boundary, the true area fraction of intersection is found from

$$
Y=1-\exp \left(-Y^{\mathrm{e}}\right)
$$

(Note that Eq. (12) has the same form as Eq. (4).) The volume fraction of transformed phase originating from nuclei on the single grain boundary can be found by integrating the area fraction of intersection over all values of the perpendicular distance $y$ between the plane and the boundary:

$$
X^{\mathrm{b}}=2 \int_{0}^{\infty} O_{\mathrm{v}}^{\mathrm{b}} Y \mathrm{~d} y=2 O_{\mathrm{v}}^{\mathrm{b}} \int_{0}^{G t}\left[1-\exp \left(-Y^{\mathrm{e}}\right)\right] \mathrm{d} y
$$

where $O_{\mathrm{v}}^{\mathrm{b}}$ is the boundary area per unit volume and the superscript on $X^{\mathrm{b}}$ indicates that the result is for nucleation on a single planar boundary. The change in the integration limits accounts for the fact that $Y^{\mathrm{e}}=0$ for $t<y / G$. The next step is to assume that the original untransformed volume contains a large number of randomly distributed grain boundaries with total area per unit volume $O_{\mathrm{v}}^{\mathrm{B}}$. When $O_{\mathrm{v}}^{\mathrm{b}}$ is replaced with $O_{\mathrm{v}}^{\mathrm{B}}$ in Eq. (13), the transformed volume fraction becomes an extended volume

${ }^{\S}$ Note that Eq. (11) is reproduced in Christian ${ }^{22}$ with a sign error on the last term in the square brackets. fraction, because impingement from regions originating on different boundaries is not yet accounted for. Because the boundaries are randomly distributed within the volume, Eq. (4) now applies. Combining Eqs. (4) and (13) gives the true transformed volume fraction for this type of process:

$$
X=1-\exp \left[-2 O_{\mathrm{v}}^{\mathrm{B}} \int_{0}^{G t}\left(1-\exp \left(-Y^{\mathrm{e}}\right)\right) \mathrm{d} y\right]
$$

Determining $X$ from Eq. (14) requires using the expression for $Y^{\mathrm{e}}$ given in Eq. (11) and performing the integration over $y$. The integration must be performed numerically, as must the differentiation of Eq. (14) with respect to time in order to obtain a transformation rate.

The transformed volume fraction depends on $G, I_{\mathrm{B}}$, and $O_{\mathrm{v}}^{\mathrm{B}}$. However, these three parameters are covariant, such that Eq. (14) has only two degrees of freedom. It is therefore proposed here that the kinetics of a boundary-nucleated process be described in terms of two independent rate constants, each with units of inverse time:

$$
\begin{aligned}
& k_{\mathrm{B}}=\left(I_{\mathrm{B}} O_{\mathrm{v}}^{\mathrm{B}}\right)^{1 / 4} G^{3 / 4} \\
& k_{\mathrm{G}}=O_{\mathrm{v}}^{\mathrm{B}} G
\end{aligned}
$$

Note that $I_{\mathrm{B}} O_{\mathrm{v}}^{\mathrm{B}}=I_{\mathrm{v}}$, and so $k_{\mathrm{B}}$ can be related directly to the rate constant $k_{\mathrm{avr}}$ in the standard Avrami equation. The rate constants $k_{\mathrm{B}}$ and $k_{\mathrm{G}}$ are associated with two distinct physical processes that occur during a boundary-nucleated transformation: $k_{\mathrm{B}}$ describes the rate at which the nucleated boundary area transforms, while $k_{\mathrm{G}}$ describes the rate at which the nonnucleated "grains" between the boundaries transform. The ratio of the two rate constants, which is unitless, determines the type of kinetic behavior that will be observed:

$$
\frac{k_{\mathrm{B}}}{k_{\mathrm{G}}}=\frac{\left(I_{\mathrm{B}} / G\right)^{1 / 4}}{\left(O_{\mathrm{v}}^{\mathrm{B}}\right)^{3 / 4}}
$$

If $O_{\mathrm{v}}^{\mathrm{B}}$ is small and $I_{\mathrm{B}} / G$ is large, such that $k_{\mathrm{B}} \gg k_{\mathrm{G}}$, then the boundary region will be densely populated with nuclei and will transform completely early in the overall process. In this case, most of the transformation will occur by the subsequent thickening of slab-like regions of transformed product centered on the original boundaries. Under these conditions, at later times, Eq. (14) approaches ${ }^{17}$

$$
X=1-\exp \left[-2 k_{\mathrm{G}} t\right]
$$

and the transformation rate decreases exponentially with time.

If $O_{\mathrm{v}}^{\mathrm{B}}$ is large and $I_{\mathrm{B}} / G$ is small, such that $k_{\mathrm{B}} \ll k_{\mathrm{G}}$, then the internal boundaries will be sparsely populated with nuclei and will transform at essentially the same rate as the entire system. In this case, restriction of the nucleation process to the boundaries is irrelevant to the kinetics, and the process meets the condition of spatially random nucleation required for the standard Avrami equation, and Eq. (14) reduces to Eq. (5) with a rate constant $k_{\mathrm{avr}}=(\pi / 3)^{1 / 4} k_{\mathrm{B}}$. Figure 2 shows the shape of the rate curve for a boundary-nucleated process, $\mathrm{d} X / \mathrm{d} t$, obtained by differentiating Eq. (14) for different values of $k_{\mathrm{B}} / k_{\mathrm{G}}$. For $\mathrm{C}_{3} \mathrm{~S}$ hydration, fitted values of $k_{\mathrm{B}} / k_{\mathrm{G}}$ are close to 1 (see Table I), indicating that neither of these limiting cases apply.

The boundary nucleation and growth model described here is in good general agreement with the results of Garrault and Nonat, ${ }^{6}$ who studied the hydration of $\mathrm{C}_{3} \mathrm{~S}$ particles in dilute suspensions with fixed $\mathrm{CaO}$ concentrations. They concluded that the early hydration period begins with the formation of $\mathrm{C}-\mathrm{S}-\mathrm{H}$ nuclei on the particle surfaces, which then grow by three-dimensional aggregation of oriented $\mathrm{C}-\mathrm{S}-\mathrm{H}$ units until the 


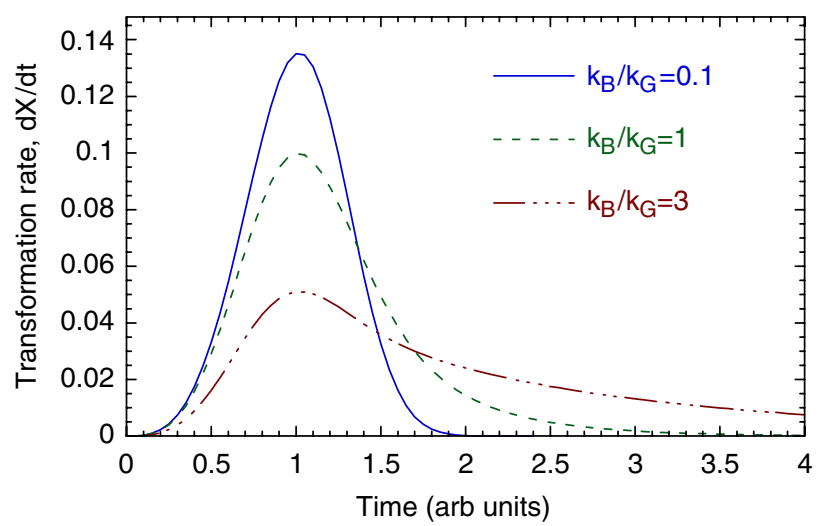

Fig. 2. Theoretical transformation rates for boundary-nucleated transformations, obtained by differentiating Eq. (14). When $k_{\mathrm{B}} / k_{\mathrm{G}}$ is small, the process approaches the conditions of spatially random nucleation specified by the standard Avrami equation. When $k_{\mathrm{B}} / k_{\mathrm{G}}$ is large, the boundary region transforms quickly and the rate decays slowly as $\exp \left(-2 k_{\mathrm{G}} t\right)$ for most of the transformation. When $k_{\mathrm{B}} / k_{\mathrm{G}}$ is close to 1 , neither process dominates.

particle surfaces are completely covered, at which point diffusion-controlled kinetics begin. They found that the hydration rate was a strong function of the rate of formation of nuclei that formed at early times, as is predicted by the process discussed in this section. One difference is that Garroult and Nonat fit their data with a model that assumed different growth rates parallel and perpendicular to the particle surface (with the former greater than the latter), while the present process assumes an equal growth rate in all directions.

\section{Experimental Procedure}

Pure triclinic $\mathrm{C}_{3} \mathrm{~S}$ powder was supplied by the Lafarge Research Center in Lyon, France. The median particle diameter was $9 \mu \mathrm{m}$ as measured by laser particle size analysis. The specific surface area as measured by small-angle neutron scattering (SANS) was $0.61 \mathrm{~m}^{2} / \mathrm{g}$. To measure the hydration kinetics, the powder was mixed by hand with deionized water for $2 \mathrm{~min}$ at a water/cement ratio of 0.5 by mass, and $\sim 0.8 \mathrm{~g}$ of paste was then tightly sealed inside a $1.3-\mathrm{mL}$ glass ampoule before being placed into the isothermal calorimeter (Model 4200, Calorimetry Sciences Corporation, Linden, UT). This instrument has a sensitivity of $0.4 \mu \mathrm{J}$ and a baseline stability of $\pm 0.08 \mu \mathrm{W} / \mathrm{h}$. Runs were conducted at temperatures ranging from $10^{\circ}$ to $40^{\circ} \mathrm{C}$. Samples were placed into the calorimeter $\sim 5 \mathrm{~min}$ after initial mixing, and another $\sim 10$ min were required for the sample temperature to become completely equilibrated to the instrument. Thus, data from the first $\sim 15$ min after mixing were not included in the nucleation and growth fits.

\section{Results and Discussion}

\section{(1) Fitting $C_{3} S$ hydration Data to the Nucleation and Growth Models}

To apply the boundary nucleation model to thermal calorimetry data from $\mathrm{C}_{3} \mathrm{~S}$ hydration, the rate of heat evolution was defined

Table I. Fit Parameters Obtained from Application of the Equations for a Boundary Nucleation Process (Eqs. (11) and (14)) to $\mathrm{C}_{3} \mathrm{~S}$ Hydration Data

\begin{tabular}{lccccc}
\hline Temperature $\left({ }^{\circ} \mathrm{C}\right)$ & $A(\mathrm{~kJ} / \mathrm{mol})$ & $k_{\mathrm{B}}\left(\mathrm{h}^{-1}\right)$ & $k_{\mathrm{G}}\left(\mathrm{h}^{-1}\right)$ & $k_{\mathrm{B}} / k_{\mathrm{G}}$ & $t_{\text {dev }} / t_{\text {peak }}$ \\
\hline 10 & 45.24 & 0.04194 & 0.04746 & 0.884 & 1.39 \\
20 & 37.68 & 0.08749 & 0.07423 & 1.179 & 1.86 \\
30 & 33.74 & 0.15034 & 0.11431 & 1.315 & 2.13 \\
40 & 34.19 & 0.22024 & 0.16559 & 1.330 & 2.18 \\
\hline
\end{tabular}

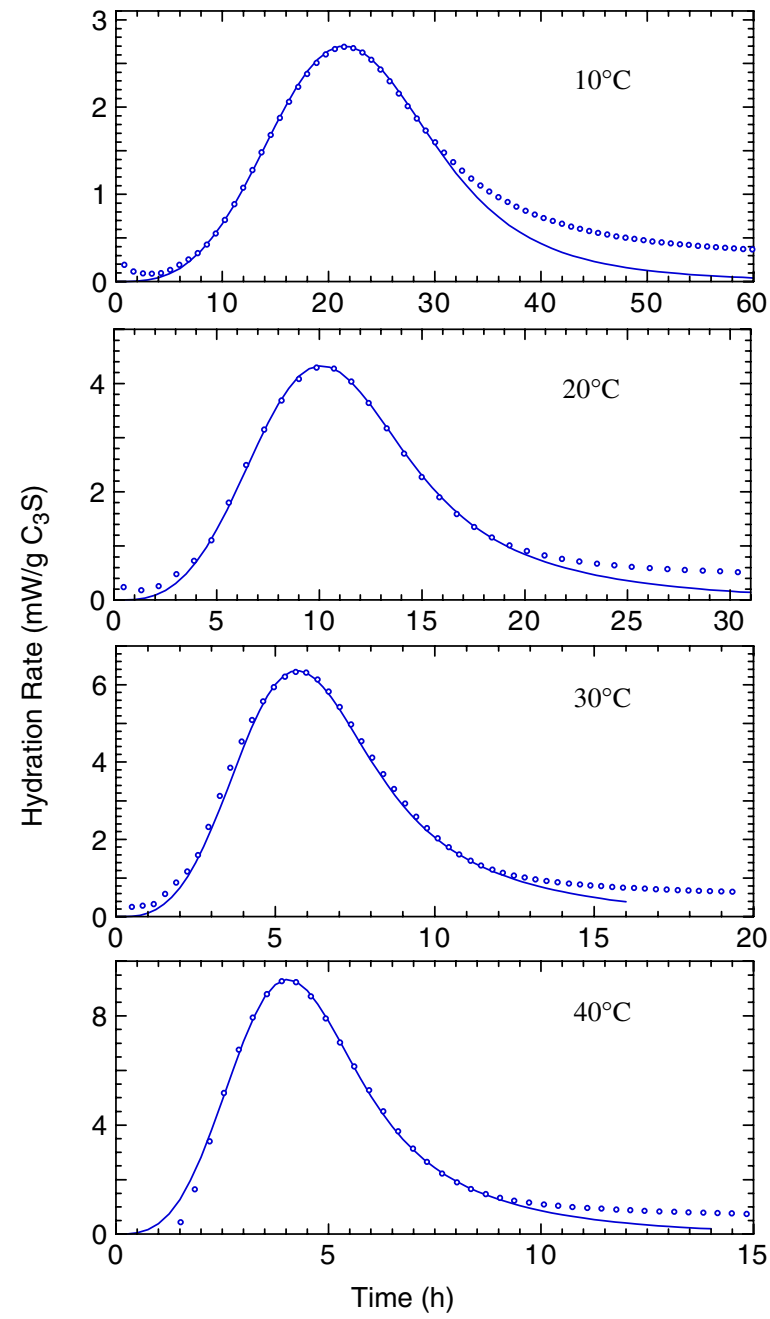

Fig. 3. Isothermal calorimetry data at different temperatures for $\mathrm{C}_{3} \mathrm{~S}$ hydration $(\mathrm{O})$ and corresponding fits using the equations derived for a boundary-nucleated nucleation and growth process (solid lines). To allow data and fits to be compared, only a fraction of the calorimetry data is shown.

as Rate $=A \mathrm{~d} X / \mathrm{d} t$, where $X$ is defined by Eqs. (11) and (14), and $A$ is a scaling parameter as defined previously for Eq. (9). The parameter $t_{0}$, corresponding to a delayed start to the nucleation and growth process (see Eq. (9)), was found to be unnecessary (this is discussed further below). Therefore, these are three-parameter fits, with $A, k_{\mathrm{B}}$, and $k_{\mathrm{G}}$ varying. A simple computer program was used to numerically perform the integration in Eq. (14) and to differentiate Eq. (14) with respect to time. Fitting was performed by manually adjusting the parameters to achieve the best agreement between the kinetic data and the model in the region of the main rate peak.

The resulting fits are shown in Fig. 3. At $20^{\circ}, 30^{\circ}$, and $40^{\circ} \mathrm{C}$, the fits follow the data for the entire early hydration peak, deviating only at the start of the slow hydration period (stage 5 in Fig. 1). At $10^{\circ} \mathrm{C}$, the transition from the main rate peak to the slow reaction period is less distinct, and the deviation of the fit from the data occurs closer to the rate peak. The fit parameters are listed in Table I. The scaling parameter $A$ decreases slightly with increasing temperature, while both rate constants increase significantly with temperature, as is expected for a thermally activated reaction. The ratio $k_{\mathrm{B}} / k_{\mathrm{G}}$ also increases with temperature, indicating that the individual parameters that make up the rate constants have different temperature dependences. This is discussed in the next section. An empirical measure of the extent to which the model describes the data is the ratio $t_{\text {dev }} / t_{\text {peak }}$, where $t_{\mathrm{dev}}$ is the time when the fit deviates from the data and $t_{\text {peak }}$ is the time of the rate peak. This is also listed in Table I. 
Table II. Fit Parameters Obtained from Application of the Avrami Equation for Nucleation and Growth (Eq. (9)) to $\mathrm{C}_{3} \mathrm{~S}$ Hydration

\begin{tabular}{lcllcc}
\hline Temperature $\left({ }^{\circ} \mathrm{C}\right)$ & $A(\mathrm{~kJ} / \mathrm{mol})$ & $t_{0}(\mathrm{~h})$ & $k_{\mathrm{avr}}\left(\mathrm{h}^{-1}\right)$ & $n$ & $t_{\text {dev }} / t_{\text {peak }}$ \\
\hline 10 & 39.24 & 3.56 & 0.04824 & 2.97 & 1.24 \\
20 & 31.52 & 2.39 & 0.1060 & 2.61 & 1.33 \\
30 & 28.28 & 1.397 & 0.1859 & 2.44 & 1.35 \\
40 & 27.73 & 1.43 & 0.2962 & 2.24 & 1.22 \\
\hline
\end{tabular}

For the purposes of comparison, the $\mathrm{C}_{3} \mathrm{~S}$ hydration data were also fit using the Avrami equation (Eq. (9)). These parameters are given in Table II, and an example fit is shown in Fig. 1. At all temperatures, these fits (which have four varying parameters) deviate from the data at an earlier point than the boundary nucleation fits (which have only three varying parameters), as evidenced by the lower values of $t_{\mathrm{dev}} / t_{\text {peak }}$. This difference between the two fits is more significant at higher temperatures than at lower temperatures. As noted earlier, when the ratio $k_{\mathrm{B}} / k_{\mathrm{G}}$ is small, as is the case at lower hydration temperatures, the two nucleation and growth models converge. The value of the exponent $n$ in the standard Avrami model decreases with increasing temperature, ranging from 2.97 at $10^{\circ} \mathrm{C}$ to 2.24 at $40^{\circ} \mathrm{C}$. According to the present interpretation, the decrease in $n$ with temperature is caused not by a change in product morphology, but by an increase in the ratio $k_{\mathrm{B}} / k_{\mathrm{G}}$, which leads to a greater deviation from the conditions of random volume nucleation required by the standard Avrami equation.

The non-zero values of parameter $t_{0}$ resulting from the standard Avrami fits (see Table II), often associated with the induction period, can now be attributed to the change in the shape of the kinetic data on the downslope of the main rate peak induced by the spatially nonrandom nucleation process (see Fig. 2), and can also be linked to fitted values of the exponent $n$ that are less than the value of 4 predicted for a process with spatially random nucleation and a constant nucleation rate (see Eq. (5)). To illustrate this, Fig. 4 shows a standard Avrami fit to the data for $\mathrm{C}_{3} \mathrm{~S}$ hydration at $10^{\circ} \mathrm{C}$, for which $t_{0}$ was constrained to be zero and the data were only fit up to the time of the maximum rate. The agreement is quite good over this range, and the fitted value of the exponent is $n=3.74$, close to the theoretical value of 4 . Gartner et $a l .{ }^{1}$ also reported that data from two different studies gave $n>3$ for the first several hours of hydration.

The rate constants $k_{\mathrm{B}}$ and $k_{\mathrm{G}}$ for a boundary-nucleated process have a combined dependence on the nucleation rate $I_{\mathrm{B}}$, the linear growth rate $G$, and the boundary area per unit volume, $O_{\mathrm{v}}^{\mathrm{B}}$ (see Eqs. (11) and (14)), and thus the latter three parameters are not determined explicitly by the fits. However, parameter

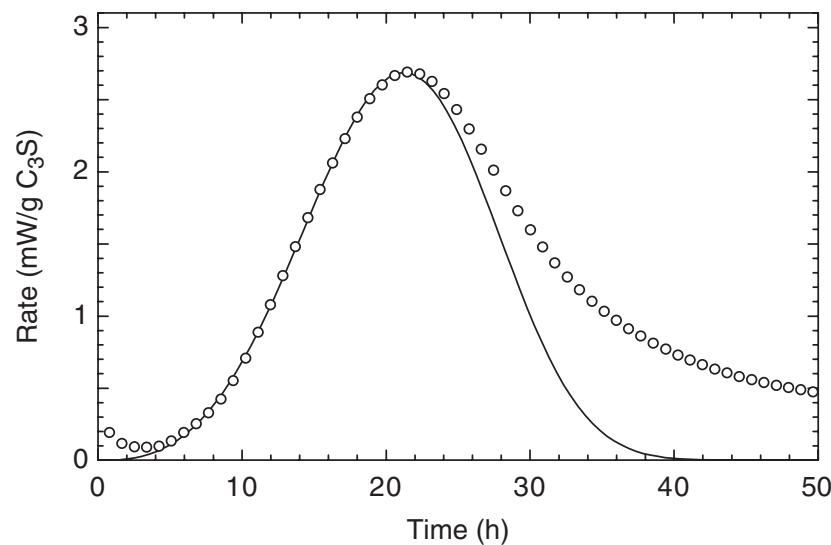

Fig. 4. The standard Avrami fit (solid line) to the $\mathrm{C}_{3} \mathrm{~S}$ hydration data at $10^{\circ} \mathrm{C}(\mathrm{O})$. The fit is based on Eq. (9), with $t_{0}$ fixed at zero, and the data were fit only up to the rate peak. The fitted value of the exponent is $n=3.74$.
Table III. Values of the Linear Growth Rate, $G$, and the Nucleation Rate, $I_{B}$, Calculated from the Rate Constants in Table I and the Independently Measured Value of $O_{\mathrm{v}}^{\mathrm{B}}=0.92 \mu \mathrm{m}^{-1}$

\begin{tabular}{lcc}
\hline Temperature $\left({ }^{\circ} \mathrm{C}\right)$ & $G(\mu \mathrm{m} / \mathrm{h})$ & $I_{\mathrm{B}}\left(\mu \mathrm{m}^{-2} \cdot \mathrm{h}\right)^{-1}$ \\
\hline 10 & 0.05159 & 0.02450 \\
20 & 0.08068 & 0.12125 \\
30 & 0.12425 & 0.28948 \\
40 & 0.17999 & 0.43859 \\
\hline
\end{tabular}

$O_{\mathrm{v}}^{\mathrm{B}}$ can be calculated by dividing the surface area of the powder $\left(0.61 \mathrm{~m}^{2} / \mathrm{g}\right.$ as obtained by SANS) by the volume occupied by the hydration products after complete hydration. From Eq. (1), using a density of $2.1 \mathrm{~g} / \mathrm{cm}^{3}$ for $\mathrm{C}-\mathrm{S}-\mathrm{H}$ (with its internal gel pores) and $2.24 \mathrm{~g} / \mathrm{cm}^{3}$ for $\mathrm{Ca}(\mathrm{OH})_{2}$, the hydration volume is $0.662 \mathrm{~cm}^{3} / \mathrm{g}$ $\mathrm{C}_{3} \mathrm{~S}$ (note that this value, and the resulting value of $O_{\mathrm{v}}^{\mathrm{B}}$, are independent of the water/cement ratio). This gives $O_{\mathrm{v}}^{\mathrm{B}}=$ $0.92 \mu \mathrm{m}^{-1}$. The resulting growth rates and nucleation rates calculated from Eq. (14) are given in Table III.

\section{(2) Temperature Dependence of the Kinetic Parameters}

Figure 5(a) shows an Arrhenius plot $(\ln k$ versus $1 / T)$ for the rate constants listed in Tables I and II. The Arrhenius plot of $k_{\mathrm{G}}$ is linear across the full temperature range sampled, with a slope that gives a constant activation energy of $31.2 \mathrm{~kJ} / \mathrm{mol}$. The Arrhenius plot for $k_{\mathrm{B}}$ is not linear, but has a slope that decreases with increasing temperature. Figure 5(b) shows a similar plot for the nucleation rate and growth rate values calculated using the measured $\mathrm{C}_{3} \mathrm{~S}$ powder surface area and listed in Table III. The growth rate shows the same linear temperature dependence as $k_{\mathrm{G}}$, indicating that it represents a single thermally activated process, while the nucleation rate increases more strongly at lower temperatures.

The values of the rate constant $k_{\mathrm{avr}}$ listed in Table II are also plotted in Fig. 5(a). This plot is reasonably linear in the temperature range of $20^{\circ}$ to $40^{\circ} \mathrm{C}$, with an apparent activation energy of $39.5 \mathrm{~kJ} / \mathrm{mol}$. This value is well within the rather wide range of previously reported values for the pure $\mathrm{C}_{3} \mathrm{~S}$ and water system over this temperature range. ${ }^{7-12}$ However, the value for $10^{\circ} \mathrm{C}$ does not follow the same trend, a phenomenon also noted in another study. ${ }^{15}$ The rate constants $k_{\mathrm{B}}$ and $k_{\mathrm{avr}}$ have similar temperature dependences, and the deviation from linearity in both cases can be attributed to their dependence on the nucleation rate, which itself has a strongly nonlinear temperature dependence.

\section{(3) Effects of Water/Cement Ratio and Particle Size}

Two problems with the application of the standard Avrami nucleation and growth model are its inability to account correctly for the kinetic effects of the water/cement ratio (w/c) of the paste or the particle size of the $\mathrm{C}_{3} \mathrm{~S}$. With the Avrami model, a change in $\mathrm{w} / \mathrm{c}$ would affect the average spacing between nuclei, such that an increase in w/c should increase the amount of hydration during the early rate peak. However, the $\mathrm{w} / \mathrm{c}$ is observed to have very little effect on the kinetics (see Fig. 6). In fact, the same basic kinetic profile for $\mathrm{C}_{3} \mathrm{~S}$ hydration is observed even in very dilute suspensions. ${ }^{6,16}$ This is predicted by the boundary nucleation model, as the average spacing between the nuclei is not affected by the $\mathrm{w} / \mathrm{c}$ because they are constrained to form only on the particle surfaces. It should be noted that at very high $\mathrm{w} / \mathrm{c}$, the assumption that the internal boundaries are randomly distributed within the transforming volume may not hold. However, this would tend to affect the kinetics only later in the reaction, when $\mathrm{C}_{3} \mathrm{~S}$ hydration is occurring under diffusion control anyway.

The situation with the particle size is reversed: the Avrami model has no sensitivity to particle size, while it is well established that the particle size has a strong effect on the kinetics, ${ }^{14,16}$ 

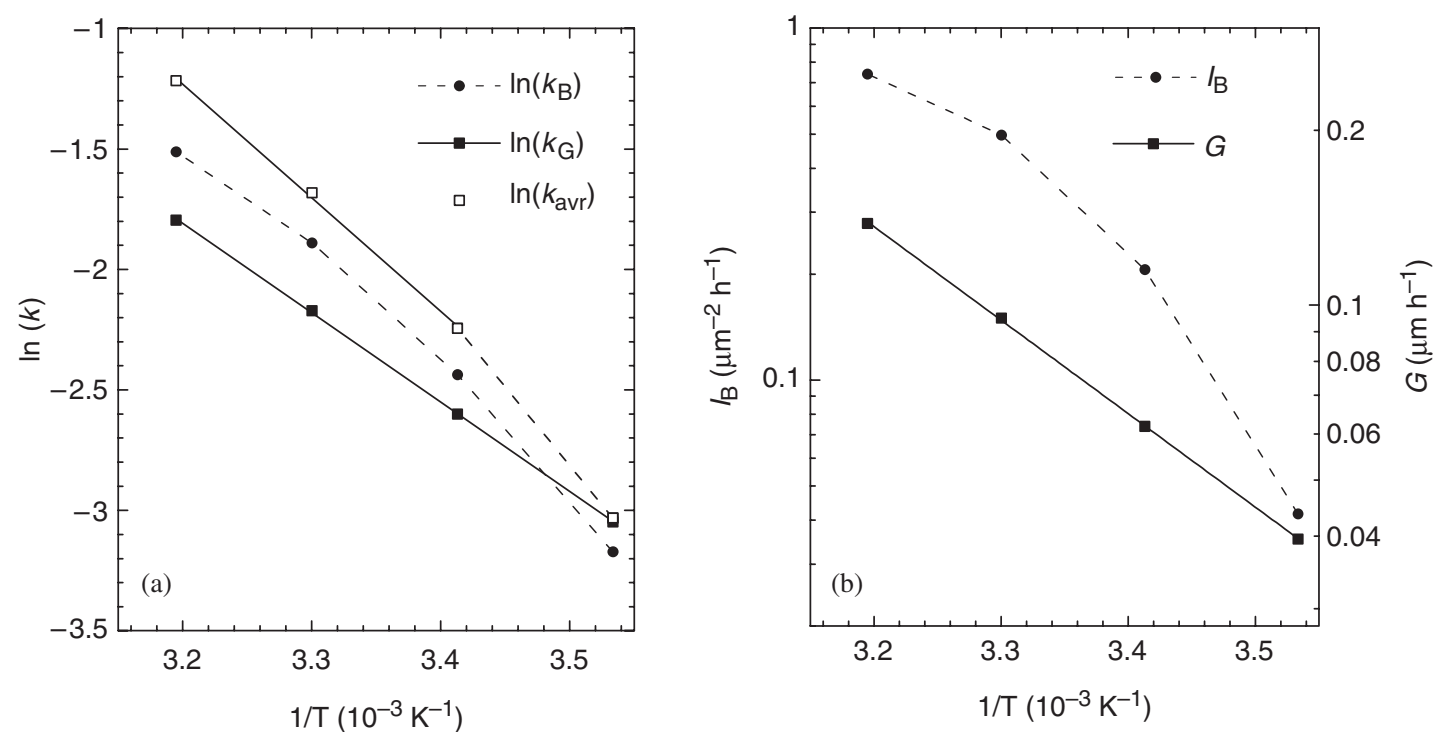

Fig. 5. The Arrhenius plot showing the temperature dependence of the fitted parameters. The dotted lines connect the data as guides to the eye and the solid lines are straight-line fits. (a) Rate constants. (b) Nucleation rate and growth rate derived from the boundary nucleation and growth fits.

with finer particles giving more hydration during the early nucleation and growth period. For example, Allen et al. ${ }^{14}$ found that the parameter $A$ for a standard Avrami fit more than doubled when the mean $\mathrm{C}_{3} \mathrm{~S}$ particle size decreased from 9.7 to 3.5 $\mu \mathrm{m}$. This effect is accounted for implicitly by the boundary nucleation process. Assuming that the transition to diffusion-controlled kinetics occurs when the layer of hydration product reaches a certain critical thickness, then the amount of nucleation and growth reaction should scale roughly with the surface area of the powder, assuming that the growth rate and nucleation rate are unchanged. This may not be the case, however, as the nucleation rate may decrease with decreasing particle size. ${ }^{16}$ The effects of particle size will be the subject of a future investigation.

\section{(4) Implications for the Induction Period}

An important finding from the boundary nucleation fits is that the time of the start of the nucleation and growth process coincides at least approximately with the time of mixing $\left(t_{0}=0\right)$. This implies that the "induction period" at the start of the reaction (stage 2 in Fig. 1), during which little hydration occurs, is not a separate chemical process, but is simply a period when the overall hydration rate is low because few nuclei have formed. A similar hypothesis was made by Taylor, ${ }^{5}$ based on the observa-

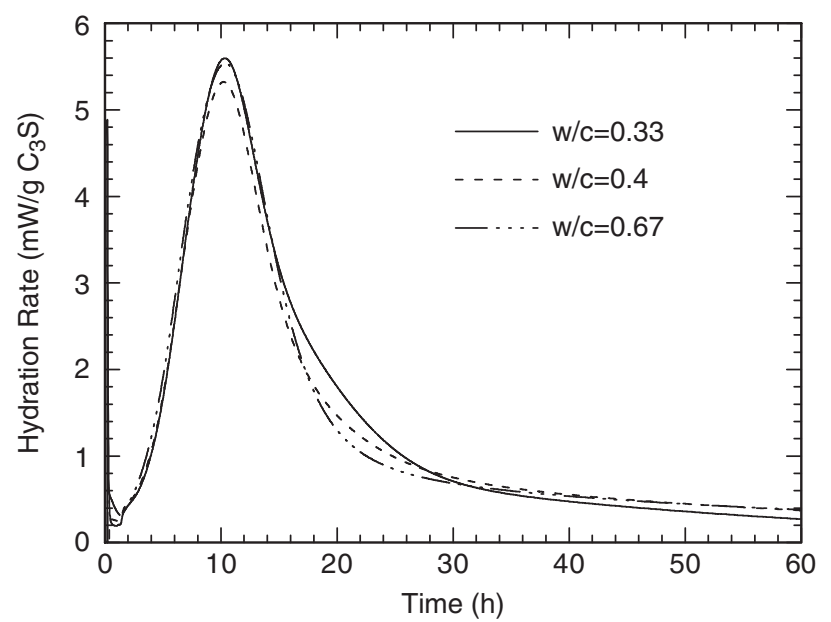

Fig. 6. Effect of the water/cement ratio (w/c) on the measured hydration kinetics of $\mathrm{C}_{3} \mathrm{~S}$. tion of Gartner and Gaidis ${ }^{4}$ that the rate of heat evolution from a $\mathrm{C}_{3} \mathrm{~S}$ paste begins to increase (albeit slowly) immediately after the end of stage 1 , and the same conclusion was also reached by Garrault and Nonat ${ }^{6}$ following a detailed study of $\mathrm{C}_{3} \mathrm{~S}$ hydration in dilute suspensions. Thus, stages $2-4$ in Fig. 1 are all controlled by the same process of boundary nucleation and growth. It should be noted, however, that the use of retarding chemical admixtures such as sucrose results in a true induction period that significantly delays the start of the accelerating reaction, presumably because initial nucleation is inhibited. ${ }^{5,28}$ The present results do not resolve the issue of whether a protective layer forms during stage 1 , because nucleation of $\mathrm{C}-\mathrm{S}-\mathrm{H}$ gel could occur either within a previously formed layer, as suggested by Gartner and Gaidis, ${ }^{4}$ or directly on the $\mathrm{C}_{3} \mathrm{~S}$ surface, as suggested by Garrault and Nonat. ${ }^{6}$

\section{Conclusions}

The equations describing the nucleation and growth kinetics of a transformation that is nucleated exclusively on randomly distributed internal boundaries, derived originally by $\mathrm{Cahn}^{17}$ to describe a solid phase transformation in a polycrystalline material, have been shown here to provide a better description of the early $\mathrm{C}_{3} \mathrm{~S}$ hydration kinetics than does the standard Avrami nucleation and growth equation. According to the boundary nucleation model, hydration product nucleates at randomly spaced locations on the surface of the $\mathrm{C}_{3} \mathrm{~S}$ particles and then grows to cover the particle surfaces and fill the capillary pores between the particles. This is in good agreement with independent observations of the hydration process.

In addition to a global scaling parameter $A$ that describes the total extent of the nucleation and growth reaction, the kinetics of a boundary nucleation process can be described by two independent rate constants introduced here for the first time: $k_{\mathrm{B}}$ describes the rate at which the nucleated boundary region transforms, while $k_{\mathrm{G}}$ describes the rate at which the nonnucleated "grains" between the boundary regions transform. These rate constants depend in turn upon the amount of boundary area (directly related to the particle size), the nucleation rate on the boundary, and the linear growth rate of product.

For the early hydration of $\mathrm{C}_{3} \mathrm{~S}$ in the temperature range of $10^{\circ}-40^{\circ} \mathrm{C}, k_{\mathrm{G}}$ exhibits a simple Arrhenius dependence with an activation energy of $31.2 \mathrm{~kJ} / \mathrm{mol}$. As the amount of boundary area is independent of temperature, this temperature dependence can also be assigned to the linear growth rate of hydration product, $G$. Rate constant $k_{\mathrm{B}}$ exhibits nonlinear Arrhenius 
behavior that can be ascribed to the behavior of the nucleation rate, $I_{\mathrm{B}}$. The nucleation rate increases rapidly with temperature at lower temperatures, but levels off at higher temperatures.

In fitting the $\mathrm{C}_{3} \mathrm{~S}$ hydration data, it was found that the time of start of the boundary nucleation process coincided with the time of mixing. This is evidence that the "induction period" at the start of the hydration reaction is a natural consequence of a nucleation and growth process with a constant nucleation rate and not a separate chemical process as is suggested by the application of the standard Avrami equation to the same data.

\section{Acknowledgments}

The author would like to thank Hamlin Jennings for useful discussions and Jeffrey Bullard for critically reading the manuscript.

\section{References}

${ }^{1}$ E. M. Gartner, J. F. Young, D. A. Damidot, and I. Jawed, "Hydration of Portland Cement"; pp. 57-113 in Structure and Performance of Cements (Chapter 3), 2nd edition, Edited by P. Barnes and J. Bensted. Spon Press, London, 2002.

${ }^{2}$ H. M. Jennings, J. J. Thomas, J. S. Gevrenov, G. Constantinides, and F.-J. Ulm, "A Multi-Technique Investigation of the Nanoporosity of Cement Paste,' Cement Concrete Res., 37, 329-36 (2007).

${ }^{3}$ R. Kondo and M. Daimon, "Early Hydration of Tricalcium Silicate: A Solid Reaction With Induction and Acceleration Periods," J. Am. Ceram. Soc., 52, 503-8 (1969).

${ }^{4}$ E. M. Gartner and J. M. Gaidis, "Hydration Mechanisms I"; pp. 95-125 in Materials Science of Concrete, Edited by J. P. Skalny. The American Ceramic Society, Westerville, OH, 1989.

${ }^{5}$ H. F. W. Taylor, Cement Chemistry, p. 155: 2nd edition, Thomas Telford, London, 1997.

${ }^{6}$ S. Garrault and A. Nonat, "Hydrated Layer Formation on Tricalcium and Dicalcium Silicate Surfaces: Experimental Study and Numerical Simulations," Langmuir, 17, 8131-8 (2001)

${ }^{7}$ N. Tenoutasse and A. DeDonder, Silicates. Ind., 35, 301 (1970).

${ }^{8}$ P. W. Brown, J. M. Pommersheim, and G. Frohnsdorff, "A Kinetic Model for the Hydration of Tricalcium Silicate," Cement Concrete Res., 15, 35-41 (1985).

${ }^{9}$ P. Fierens, Y. Kabuema, and J. Tirlocq, "Influence of Quenching Temperature on the Hydration of $\mathrm{C}_{3} \mathrm{~S}$," Cement Concrete Res., 12, 191-8 (1982).
${ }^{10}$ S. A. FitzGerald, D. A. Neumann, J. J. Rush, D. P. Bentz, and R. A. Livingston, "In-Situ Quasielastic Neutron Scattering Study of the Hydration of Tricalcium Silicate," Chem. Mater., 10, 397-402 (1998)

${ }^{11}$ J. J. Thomas and H. M. Jennings, "Effects of $\mathrm{D}_{2} \mathrm{O}$ and Mixing on the Early Hydration Kinetics of Tricalcium Silicate," Chem. Mater., 11, 1907-14 (1999).

${ }^{12}$ D. R. Vollet and A. F. Craievich, "Effects of Temperature and of the Addition of Accelerating and Retarding Agents on the Kinetics of Hydration of Tricalcium Silicate," J. Phys. Chem. B, 104, 12143-8 (2000).

${ }^{13}$ A. Damasceni, L. Dei, E. Fratini, F. Ridi, S. H. Chen, and P. Baglioni, "A Novel Approach Based on Differential Scanning Calorimetry Applied to the Study of Tricalcium Silicate Hydration Kinetics," J. Phys. Chem. B, 106, 11572-8 (2002)

${ }^{14}$ A. J. Allen, J. C. McLaughlin, D. A. Neumann, and R. A. Livingston, "In Situ Quasi-Elastic Scattering Characterization of Particle Size Effects on the Hydration of Tricalcium Silicate," J. Mater. Res., 19, 3242-54 (2004).

${ }^{15}$ S. A. Grant, G. E. Boitnott, C. J. Korhonen, and R. S. Sletten, "Effect of Temperature on Hydration Kinetics and Polymerization of Tricalcium Silicate in Stirred Suspensions of CaO-Saturated Solutions," Cement Concrete Res., 36, 671-7 (2006).

${ }^{16}$ S. Garrault, T. Behr, and A. Nonat, "Formation of the C-S-H layer During Early Hydration of Tricalcium Silicate Grains With Different Sizes," J. Phys. Chem. B, 110, 270-5 (2006).

${ }^{17}$ J. W. Cahn, "The Kinetics of Grain Boundary Nucleated Reactions," Acta Metall., 4, 449-59 (1956)

${ }^{18}$ M. Avrami, "Kinetics of Phase Change," J. Chem. Phys., 8, 1103-12 (1939)

${ }^{19}$ M. Avrami, "Kinetics of Phase Change," J. Chem. Phys., 8, 212-24 (1940).

${ }^{20}$ A. N. Kolmogorov, "A Statistical Theory for the Recrystallization of Metals," Bull. Acad. Sci. USSR Phys. Ser., 1, 255 (1937).

${ }^{21}$ W. A. Johnson and R. F. Mehl, "Reaction Kinetics in Processes of Nucleation and Growth," Trans. Am. Inst. Min. (Metall.) Eng., 135, 416 (1939).

${ }^{22} \mathrm{~J}$. W. Christian, The Theory of Transformations in Metals and Alloys, Part 1, 3rd edition, Pergamon Press, Oxford, 2002.

${ }^{23}$ R. A. Livingston, "Fractal Nucleation and Growth Model for the Hydration of Tricalcium Silicate," Cement Concrete Res., 30, 1853-60 (2001).

${ }^{24}$ I. G. Richardson, "Tobermorite/Jennite- and Tobermorite/Calcium Hydroxide-Based Models for the Structure of C-S-H: Applicability to Hardened Pastes of Tricalcium Silicate,-Dicalcium Silicate, Portland Cement, and Blends of Portland Cement With Blast-Furnace Slag, Metakaolin, or Silica Fume," Cement Concrete Res., 34, 1733-77 (2004).

${ }^{25} \mathrm{~A}$. Nonat, "The Structure and Stoichiometry of $\mathrm{C}-\mathrm{S}-\mathrm{H}, "$ Cement Concrete Res., 34, 1521-8 (2004).

${ }^{26}$ A. J. Allen, R. C. Oberthur, D. Pearson, P. Schofield, and C. R. Wilding, "Development of the Fine Porosity and Gel Structure of Hydrating Cement Systems," Phil. Mag. B, 56, 263-8 (1987).

${ }^{27} \mathrm{G}$. Constantinides and F.-J. Ulm, "The Nanogranular Nature of C-S-H," J. Mech. Phys. Solids, 55, 64-90 (2007).

${ }^{28}$ M. Bishop, S. G. Bott, and A. R. Barron, "A New Mechanism for Cement Hydration Inhibition: Solid-State Chemistry of Calcium Nitrilotris (Methylene) Triphosphonate," Chem. Mater., 15, 3074-88 (2003). 